Positions moyennes des étoiles de comparaison.

\begin{tabular}{|c|c|c|c|c|c|c|c|}
\hline * & \multicolumn{3}{|c|}{$\alpha 1903.0$} & \multicolumn{3}{|c|}{$\delta 1903.0$} & Autorité \\
\hline $\mathbf{r}$ & I $7^{\mathrm{h}}$ & & $42 \cdot 5 \mathrm{I}$ & - I $4^{\circ}$ & 19 & 2 I" 2 & $M_{1} \quad 3_{698}$ \\
\hline 2 & I 7 & & 36.83 & -14 & 8 & 59.0 & rapp. à *3 \\
\hline 3 & I 7 & & 32.37 & $-x_{4}$ & I 2 & 30.6 & $M_{1} I 3479$ \\
\hline 4 & I 7 & & 16.84 & $-I 4$ & 13 & 50.8 & $M_{1} I 347^{2}$ \\
\hline 5 & I I & 9 & $5^{2}$ & +6 & 8 & & pos. approchée \\
\hline 6 & I I & & $50.9^{6}$ & +6 & 8 & 56.4 & AG. Leipzig II $575^{\circ}$ \\
\hline 7 & I I & & I 7.99 & +6 & 29 & 48.5 & $\gg \quad \gg 5735$ \\
\hline 8 & I I & 6 & $2 \mathrm{x} .62$ & +6 & 29 & $9 \cdot 9$ & $\searrow 5718$ \\
\hline 9 & 22 & Io & I 9.56 & $-2 I$ & 45 & 293 & Cinc. $374 I$ \\
\hline IO & 22 & 8 & 44.72 & $-2 \mathrm{I}$ & 53 & 31.5 & Cinc. 3734 \\
\hline I I & $2 \mathrm{I}$ & 56 & 47.98 & -22 & 34 & 8.0 & rapp. à Cord. GC. 30196 \\
\hline I 2 & $2 \mathrm{I}$ & $5 \mathrm{I}$ & 4.00 & -22 & 33 & 38.8 & $\mathrm{AOe}_{2} 2174^{6}$ \\
\hline I 3 & $2 \mathrm{I}$ & 52 & $54 \cdot 37$ & $-2 \mathrm{I}$ & I I & 57.5 & Cinc. 3692 \\
\hline 14 & $2 \mathrm{I}$ & 50 & I 2.33 & -20 & 52 & 46.6 & Cinc. $368 \mathrm{I}$ \\
\hline I 5 & 22 & 42 & 22.47 & -7 & $5^{6}$ & 45.0 & $M_{13572}$ \\
\hline I 6 & 22 & 39 & 31.55 & -8 & 7 & $35 \cdot 7$ & Paris 32606 \\
\hline I 7 & 22 & 4 I & 20.31 & -8 & I9 & $43 \cdot 2$ & $\mathrm{M}_{13} 3 \mathrm{I} 549$ \\
\hline I 8 & Io & 58 & $57 \cdot 35$ & +16 & 46 & $46 . I$ & AG. Berlin A. 4343 \\
\hline 19 & I 4 & 44 & 35.05 & -8 & 47 & 55.2 & $M_{1} 10605$ \\
\hline 20 & I 4 & 44 & $5^{2.7 I}$ & -8 & $3^{2}$ & $5^{6.5}$ & $M_{1} I 06 I I$ \\
\hline
\end{tabular}

\begin{tabular}{|c|c|c|c|c|c|c|c|}
\hline$*$ & \multicolumn{3}{|c|}{$\alpha 19 \circ 3.0$} & \multicolumn{3}{|c|}{$\delta 1903.0$} & Autorité \\
\hline 2 I & I 4 & 40 & $\mathrm{~m}_{5} 5 . .00$ & -8 & ${ }^{\circ} 23^{\prime}$ & $59: 6$ & rapp. à * 20 \\
\hline 22 & I 4 & 42 & 46 & -8 & I 5 & & pos. approchée \\
\hline 23 & I. 4 & 40 & I I.I 8 & -8 & 3 & 9.2 & $M_{1}$ I05IO \\
\hline 24 & I 4 & 39 & 5.68 & -7 & 50 & $33 \cdot 3$ & $\mathrm{M}_{1} \mathrm{IO}_{48}$ \\
\hline 25 & 22 & 6 & 41.24 & -10 & I3 & $25 \cdot 3$ & $M_{13} 30388$ \\
\hline 26 & & 2 & 8.88 & -9 & 39 & 22.2 & Sant. 2482 \\
\hline 27 & & 47 & 2.02 & -7 & 48 & 8.4 & $M_{1} 29694$ \\
\hline 28 & $2 \mathrm{I}$ & 46 & 26.32 & -7 & $5 \mathrm{I}$ & 30.2 & $M_{1} 29^{675}$ \\
\hline 29 & $2 I$ & 46 & 59.86 & -7 & 25 & 35.6 & $M_{1} 29693$ \\
\hline 30 & $2 \mathrm{I}$ & 42 & 31.94 & -6 & 22 & $3 \cdot 1$ & $\mathrm{M}_{1} 295$ I 5 \\
\hline $3 \mathrm{I}$ & $\circ$ & I 7 & 16.23 & $-I$ & 28 & $5^{8.6}$ & AG. Nicol. $5^{\circ}$ \\
\hline 32 & 2 & S & 54.98 & +4 & 25 & 2.2 & AG. Albany 630 \\
\hline 33 & 2 & $c$ & 30.48 & +4 & 18 & 34.8 & $\gg \quad \gg 611$ \\
\hline 34 & 2 & 6 & 57.02 & +4 & 8 & $4 \cdot 5$ & 6 I 4 \\
\hline 35 & 2 & $x$ & 30.25 & +3 & $5^{2}$ & 41.8 & $\Rightarrow \quad 593$ \\
\hline 36 & I 7 & $5^{2}$ & $24.3 \mathrm{I}$ & -23 & $4^{r}$ & $27 \cdot 3$ & Cord. GC. 24394 \\
\hline 37 & $\circ$ & 32 & 43.87 & +16 & $5 x^{x}$ & 46.7 & rapp. à AG. Berlin A. 165 \\
\hline $3^{8}$ & I & 51 & 37.73 & $+\cdot 8$ & 59 & 20.5 & AG. Leipzig II 740 \\
\hline 39 & r & $4 C$ & 2.52 & +9 & 7 & $2 \mathrm{I} .8$ & $\gg \quad \gg 723$ \\
\hline
\end{tabular}

Remarques.

(I 7) Thetis. I9 mai. Les images sont très agitées. (184) Dejopeja. 3 I août. Une étoile de $I 2^{\ominus} \mathrm{gr}$., très voisine et sur le même parallèle que la planète, contrarie les pointés. - 2 sept. Le ciel étant nébuleux, l'éclat de la planète est d'une faiblesse extrême.
(258) Tyche. I5 mai. La planète disparaît sous d'épais nuages. - 18 mai. Le ciel est très nébuleux. 25 mai. Un fort vent du $\mathrm{NE}$ secoue la lunette.

$(362)$ Havnia. 20 octobre. Sur la fin de l'observation le ciel se couvre de cirrus.

\title{
Ephemeride des Planeten (505) [1902 LL] = 1904 NA.
}

$\mathrm{I}^{\mathrm{h}}$ M. Z. Berlin.

\begin{tabular}{|c|c|c|c|c|}
\hline 1904 & $\alpha \mathrm{I} 904.0$ & $\delta 1904.0$ & $\log r$ & $\log A$ \\
\hline Febr. 27 & $5^{h} 12^{m} 40^{s}$ & $+26^{\circ} \times 7: 8$ & 0.3278 & 0.2296 \\
\hline 29 & I 5 I I & $\begin{array}{ll}26 & 26.3\end{array}$ & & \\
\hline März 2 & 1746 & $26 \quad 34.6$ & 0.3292 & 0.2425 \\
\hline 4 & 2027 & 2642.5 & & \\
\hline 6 & $23 \times 3$ & $26 \quad 50.2$ & 0.3307 & $0.255^{2}$ \\
\hline 8 & 263 & $26 \quad 57.5$ & & \\
\hline 10 & $285^{8}$ & $27 \quad 4.6$ & $0.33^{22}$ & 0.2676 \\
\hline I 2 & 3157 & $27 \quad 113$ & & \\
\hline 14 & 35 I & $27 \quad 17.6$ & 0.3337 & $0.279^{8}$ \\
\hline 16 & 388 & 2723.6 & & \\
\hline I 8 & 4 I I 9 & $27 \quad 29.3$ & $0.335^{2}$ & 0.2917 \\
\hline 20 & $44 \quad 34$ & $27 \quad 34.7$ & & \\
\hline 22 & 4753 & 2739.6 & $0.33^{68}$ & 0.3033 \\
\hline 24 & $5^{\text {I }} \quad$ I 4 & $27 \quad 44.2$ & & \\
\hline 26 & $\begin{array}{lll}5 & 54 & 39\end{array}$ & +2748.4 & $0.33^{84}$ & 0.3147 \\
\hline
\end{tabular}

\begin{tabular}{|c|c|c|c|c|}
\hline 1904 & $\alpha 1904.0$ & $\delta 1904.0$ & $\log r$ & $\log \Delta$ \\
\hline $\begin{array}{r}\text { März } 26 \\
28\end{array}$ & $\begin{array}{llr}5^{\mathrm{h}} & 54^{\mathrm{m}} 39^{\mathrm{s}} \\
5 & 58 & 6\end{array}$ & $\begin{array}{r}+27^{\circ} 48: 4 \\
2752.2\end{array}$ & 0.3384 & 0.3147 \\
\hline 30 & $6 \quad 37$ & 2755.6 & 0.3400 & 0.3257 \\
\hline April $\mathbf{I}$ & 510 & 2758.6 & & \\
\hline 3 & 846 & $28 \quad 1.2$ & $0.34^{16}$ & $0.33^{64}$ \\
\hline 5 & I 224 & 3.4 & & \\
\hline 7 & I 64 & 5.2 & 0.3433 & 0.3469 \\
\hline 9 & 1947 & 6.6 & & \\
\hline I I & 2331 & $7 \cdot 5$ & $0.345^{\circ}$ & $0.357 \mathrm{I}$ \\
\hline I 3 & $27 \quad 17$ & 8.0 & & \\
\hline I 5 & 3 I 5 & 8. 1 & 0.3467 & $0.3^{6} 7^{\circ}$ \\
\hline 17 & 3455 & $7 \cdot 7$ & & \\
\hline I 9 & 3846 & 7.0 & 0.3484 & 0.3766 \\
\hline 2 I & $42 \quad 39$ & 5.8 & & \\
\hline 23 & $\begin{array}{lll}6 & 46 & 33\end{array}$ & +28 & 0.3502 & 0.3859 \\
\hline
\end{tabular}

Der Ephemeride liegen die Elemente von $(5 \circ 5)$ in freundlichst zur Verfügung gestellt hat. Eine Beobachtung A. N. 3908 zugrunde, jedoch mit einer zur Darstellung der Beobachtungen von 1904 NA erforderlichen Verbesserung $\Delta M=+$ Ir:24, die Prof. A. Berberich ermittelt und mir

Bremen, I 904 Februar.

Wien Febr. 19 ergibt als Korrektion obiger Ephemeride $(\mathrm{B}-\mathrm{R})$ :

$$
\Delta \alpha=-6^{\mathrm{s}} \quad \Delta \delta=- \text { - }: 0 .
$$

Hans Osten. 